\title{
RUNX1 inhibits the antiviral immune response against influenza $A$ virus through attenuating type I interferon signaling
}

\author{
Yixiang Hu ${ }^{1,3+}$, Qi Pan ${ }^{1+}$, Kun Zhou' ${ }^{1}$, Yuehuan Ling ${ }^{1}$, Hao Wang ${ }^{1}$ and Yan $\mathrm{Li}^{1,2,3^{*}+}$ (i)
}

\begin{abstract}
Background: Influenza A viruses (IAVS) are zoonotic, segmented negative-stranded RNA viruses. The rapid mutation of IAVs results in host immune response escape and antiviral drug and vaccine resistance. RUNX 1 is a transcription factor that not only plays essential roles in hematopoiesis, but also functions as a regulator in inflammation. However, its role in the innate immunity to IAV infection has not been well studied.

Methods: To investigate the effects of RUNX1 on IAV infection and explore the mechanisms that RUNX1 uses during IAV infection. We infected the human alveolar epithelial cell line (A549) with influenza virus A/Puerto Rico/8/34 (H1N1) (PR8) and examined RUNX1 expression by Western blot and qRT-PCR. We also knocked down or overexpressed RUNX1 in A549 cells, then evaluated viral replication by Western blot, qRT-PCR, and viral titration.

Results: We found RUNX1 expression is induced by IAV H1N1 PR8 infection, but not by poly(l:C) treatment, in the human alveolar epithelial cell line A549. Knockdown of RUNX1 significantly inhibited IAV infection. Conversely, overexpression of RUNX1 efficiently promoted production of progeny viruses. Additionally, RUNX1 knockdown increased IFN- $\beta$ and ISGs production while RUNX1 overexpression compromised IFN- $\beta$ and ISGs production upon PR8 infection in A549 cells. We further showed that RUNX1 may attenuate the interferon signaling transduction by hampering the expression of IRF3 and STAT1 during IAV infection.
\end{abstract}

Conclusions: Taken together, we found RUNX1 attenuates type I interferon signaling to facilitate IAV infection in A549 cells.

Keywords: Influenza A virus, RUNX1, IFN, IRF3, STAT1, A549

\section{Introduction}

The Influenza A viruses (IAVs) are enveloped viruses within the family Orthomyxoviridae. The genome of IAVs, which are composed of eight segmented negative-stranded RNA (PB2, PB1, PA, HA, NA, NP, M, and NS), encode at least 17 proteins [1]. IAVs are contagious

\footnotetext{
*Correspondence: yanli3@zju.edu.cn

${ }^{\dagger}$ Yixiang Hu, Qi Pan and Yan Li have contributed equally to this work. ${ }^{1}$ Department of Veterinary Medicine and Institute of Preventive Veterinary Sciences, Zhejiang University College of Animal Sciences, Hangzhou 310058, Zhejiang, China

Full list of author information is available at the end of the article
}

pathogens that can infect humans and a variety of animals, such as swine, poultry, equine, canine, and bats. In humans, IAVs primarily infect and replicate in epithelial cells of respiratory tract, resulting in alveolar epithelial cell injury and causes mild to severe pneumonia. Occasionally, IAV infection is associated with secondary bacterial infections, which may lead to fatal outcomes [2, 3].

During viral infection, the innate immune system is the host's first line of defense against virus invasion [4]. Once IAV invades, viral pathogen-associated molecular patterns (PAMPs), such as viral RNAs and intermediate RNAs, are quickly recognized by the host through pathogen recognition receptors (PRRs), which include 
Toll-like receptors (TLRs), retinoic acid-inducible gene I (RIG-I)-like receptors, and nucleotide-binding oligomerization domain-like receptors [5]. Recognition of PAMPs by PPRs leads to innate immune signaling activation and transcriptional activity increase by transcription factors, such as IFN regulatory factor 3 (IRF3), nuclear factor kappa B (NF-kB), activator protein 1(AP1), and the coactivator CREB binding protein (CBP) and/or p300 [4]. Among these transcription factors, IRF3 is one of the most essential ones for interferon $\beta$ (IFN- $\beta$ ) production. Upon viral infection, phosphorylated IRF3 forms complex with co-activator $\mathrm{CBP} / \mathrm{p} 300$, then the complex translocates to the nucleus and binds the regulatory domains of the IFN- $\beta$ promoter to initiate transcription of IFN- $\beta$ [6]. IFN- $\beta$, which belongs to type I IFNs, is one of the key antiviral cytokines produced by influenza A virusinfected epithelial cells. The antiviral activity of type I IFN is mediated by a set of interferon-stimulated genes (ISGs) $[7,8]$. Binding of IFN- $\beta$ to its receptor is the initial step in this signaling process, followed by activation of the JAK family and subsequent activation of STAT1 protein that finally induces the production of ISGs which target different steps of the IAV life cycle $[4,9,10]$.

The RUNX family consists of RUNX1, RUNX2, and RUNX3. In humans, RUNX1 is identified as one of the genes most frequently altered by chromosome translocation and point mutations in acute myelogenous leukemia (AML) $[11,12]$, so RUNX1 is also known as AML1. As a transcription factor, Runx1 has critical roles in embryonic and cell development, hematopoiesis, angiogenesis, tumorigenesis $[13,14]$. Also, RUNX1 functions as a regulator in the inflammatory signaling. It suppresses LPS-induced inflammatory response in pulmonary diseases by inhibiting the NF- $\mathrm{KB}$ signaling pathway [15]. It also attenuates NF- $\mathrm{kB}$ signaling in myeloid cells through interaction with IкB kinase complex in the cytoplasm, so the AML1 mutant related leukemia cell exhibits distinctly activated NF- $\mathrm{B}$ signaling [16]. However, it interacts with the NF- $\mathrm{kB}$ subunit $\mathrm{p} 50$ to enhance NF- $\mathrm{kB}$ mediated inflammatory signaling in macrophages upon LPS stimulation [17]. So the roles for RUNX1 in the inflammatory signaling are distinct in different situations. Beyond that, RUNX1 also plays an important role in HIV-1, SARSCoV, Epstein-Barr virus, and VSV infection [18-26].

Like many other viruses, IAVs have evolved strategies to exploit and hijack the cellular machinery to escape innate immune response, especially to circumvent the type I IFN system [27]. Therefore, identifying and targeting the key inducible cellular factors that modulate IAV replication and pathogenesis would provide a potential solution to develop efficient antiviral drugs or vaccines [28-30].To identify cellular genes required in the IAV infectious cycle, several groups performed genome-wide screenings in different cells with different IAVs. Brass et al. conducted RNAi screens in osteosarcoma cells (U2OS) infected with influenza A/Puerto Rico/8/34 (H1N1) (PR8), and Karlas et al. performed RNAi screens in a human alveolar epithelial cell line (A549) with influenza A/WSN/33(H1N1) and the pandemic A/ Hamburg/04/2009(H1N1) virus infection, and both of the groups identified RUNX1 as one of the targets [31, 32], which indicated that RUNX1 might promote IAV infection. [33]. Gaur et al. identified the interaction of NA protein of H1N1 PR8 virus with RUNX1 in A549 cells, and the knockdown of RUNX1 using siRNA resulted in decreased IFN- $\beta$ expression in human myeloid leukemia cell line U937 [34]. However, the role and mechanism of RUNX1 in IAV H1N1 PR8 virus infection in A549 cells is not further studied.

Here, we investigated the effects and mechanisms of RUNX1 on IAV PR8 infection by both gain- and loss-offunction studies. We found that RUNX1 is induced by IAV PR8 infection in the A549 cells. The knockdown of RUNX1 significantly inhibited IAV infection, while overexpression of RUNX1 promoted IAV infection. Moreover, induction of RUNX1 inhibited the expression of IFN signaling related proteins IRF3 and STAT1, and influenced the production of IFN- $\beta$ and ISGs. Our results demonstrated that RUNX1, acting as a negative regulator of the IFN signaling pathway, is capable of attenuating antiviral defenses and facilitating IAV infection.

\section{Materials and methods \\ Cell culture and virus}

Human alveolar adenocarcinoma epithelial (A549) cells, human embryonic kidney (HEK 293T) cells, and MadinDarby canine kidney (MDCK) cells were used in this study. A549 cells were maintained in the RPMI 1640 (Thermo Fisher, Waltham, MA, USA) plus 10\% fetal bovine serum (FBS) (ExCell Biology, shanghai, China). 293T cells were cultured in the Dulbecco's modified Eagle's medium (DMEM) (Thermo Fisher, Waltham, MA, USA) supplemented with $10 \%$ FBS and $100 \mathrm{U} / \mathrm{mL}$ penicillin and $0.1 \mathrm{mg} / \mathrm{mL}$ streptomycin (Thermo Fisher, Waltham, MA, USA). The MDCK cells were grown in minimum essential medium (MEM) (Thermo Fisher, Waltham, MA, USA) containing 10\% FBS, $1 \mathrm{mM}$ sodium pyruvate (Thermo Fisher, Waltham, MA, USA), 100U/ $\mathrm{mL}$ penicillin, and $0.1 \mathrm{mg} / \mathrm{mL}$ streptomycin.

The influenza virus A/Puerto Rico/8/34 (H1N1) (PR8), A/Zhejiang/163/2020 (H3N2) (ZJ163), A/ swine/Jiangsu/C1/2008 (H9N2) (JSC1) and A/ California/04/2009(H1N1) (CA04) was propagated in 10-day-old chicken embryos, and viral titers were determined by calculating the $50 \%$ tissue culture infectious 
dose $\left(\mathrm{TCID}_{50}\right)$ per milliliter using the Reed-Muench method in MDCK cells.

\section{Antibodies}

Mouse anti-RUNX1(A-2) (sc-365644) antibodies were purchased from Santa Cruz, Dallas, TX, USA; rabbit antiIRF3 (11312-AP) and rabbit anti-STAT1 (10144-2-AP) were purchased from Proteintech, Rosemont, IL, USA; rabbit anti-P-IRF3 (Ser396) (4D4G) antibodies and rabbit anti-P-STAT1 (Tyr701) (D4A7) antibodies were purchased from Cell Signaling, Danvers, MA, USA; mouse anti-GAPDH antibody (AF0006) was purchased from Beyotime, Shanghai, China; mouse mAbs to viral proteins NP and M1 of IAV were obtained from Dr. Jiyong Zhou [35].

\section{Viral infection and $T C I D_{50}$ assay}

When the MDCK cells grew to 95\% confluent monolayers, the cell culture medium was withdrawn and the cells were washed twice with phosphate-buffered saline (PBS), and then viruses in viral growth medium were inoculated in the cells. After adsorption at $37{ }^{\circ} \mathrm{C}$ for $1 \mathrm{~h}$, the inoculum was removed and replaced with a viral growth medium. The viral growth medium was a serum-free medium supplemented with $2 \%$ BSA V and $2 \mu \mathrm{g} / \mathrm{mL}$ of tosylsulfonyl phenylalanyl chloromethyl ketone (TPCK)trypsin (Sigma, St. Louis, MO, USA).

To measure the infectious titer of virus in cell culture supernatants, MDCK cells were plated in 96-well plates and grow to $90-95 \%$ confluence overnight. The collected viral supernatants were immediately serially diluted in a serum-free medium. MDCK cells were then infected as described above. IAV-induced cytopathic effect (CPE) was monitored for $24-96 \mathrm{~h}$. TCID $_{50}$ was then calculated by the Reed-Muench formula.

\section{Vector construction and transfections}

The wild-type RUNX1 gene was obtained from the THP-1 human monocytic leukemia cell line by RT-PCR and subsequently subcloned into pCMV expression vector via $\mathrm{Xba \textrm {I }}$ and $\mathrm{BamH \textrm {I }}$ sites. Corresponding primers used were as follows: pCMV-RUNX1 CDS forward, 5'-ATG GCT TCA GAC AGC ATA TT-3', reverse, 5'-TCA GTA GGG CCT CCA CAC GG-3'. Recombinant plasmids were confirmed by DNA sequencing and plasmids were prepared by using OMEGA EndoFree Plasmid Mini Kit I (OMEGA Bio-Tek, Norcross, GA, USA). Transfection of plasmids to $80 \sim 90 \%$ confluent cell monolayers was performed using the Lipofectamine 2000 transfection reagent (Thermo Fisher, Waltham, MA, USA) according to the manufacturer's instructions. Polyinosinic-polycytidylic acid (poly (I:C))
(Tocris Bioscience, Bristol, UK) was introduced to the cells with or without Lipofectamine 2000 transfection reagent.

\section{Generation of stable cell lines with RUNX1 knockdown}

To generate the stable cell lines that have RUNX1 knocked down, A549 cells were transduced with pseudotyped lentiviruses which express RUNX1-shRNA or Luci-shRNA (Luci is short for luciferase) as control. We used the third generation of lentivirus packaging system that contains three packaging plasmids (pGag/Pol, pRev, and pVSV-G) and one expression plasmid (shRNApCD513B-1 plasmid containing Puro and GFP hU6 promoter) to produce the lentiviruses we needed. The sequences for RUNX1-shRNA and Luci-shRNA were designed by using Invitrogen online shRNA design software and synthesized and subsequently subcloned into pCD513B-1 expression vectors via SpeI and BamHI. The sequences are as follows: RUNX1-shRNA: 5'-GAA CCA GGT TGC AAG ATT TAA-3'; Luci-shRNA: 5'-CGT ACG CGG AAT ACT TCG A-3'; the loop: 5'-CTC GAG-3'. Plasmids for RUNX1shRNA or Luci-shRNA expression combined with three packaging plasmids were mixed with Lipofectamine 2000 (Thermo Fisher, Waltham, MA, USA) and transfected into HEK293T cells according to the manufacturer's protocol. The transfection medium was replaced with advanced DMEM supplemented with $2 \%$ FBS, $0.01 \mathrm{mM}$ L-a-phosphatidylcholine, $0.01 \mathrm{mM}$ cholesterol (Sigma, St. Louis, MO, USA), 4.0 mM L-glutamine, and 1:1000 diluted chemically defined lipid (Thermo Fisher, Waltham, MA, USA) at $16 \mathrm{~h}$ post-transfection. The cell-free supernatant that contains lentiviruses were collected at $48 \mathrm{~h}$ after transfection and lentiviral titers were determined by $\mathrm{TCID}_{50}$ assay in HEK293T cells. To generate A549 cells that stably express RUNX1-shRNA or Luci-shRNA, A549 cells in six-well plates were inoculated with lentivirus at a multiplicity of infection (MOI) of and $4 \mathrm{~mL}$ RPMI 1640 fresh medium plus $10 \% \mathrm{FBS}$. After incubation at $37^{\circ} \mathrm{C}$ for $12 \mathrm{~h}$, the inoculum was removed and fresh medium was added, and the cells were incubated at $37{ }^{\circ} \mathrm{C}$ for another $48 \mathrm{~h}$. The lentivirus-infected A549 cells were selected by supplementation of $2 \mathrm{~g} / \mathrm{mL}$ puromycin (Sigma, St. Louis, MO, USA) in the medium to generate the shRUNX1 or shControl A549 stable cell line. Since the shRNA expression plasmid contains a GFP reporter, the green fluorescence was detected under a fluorescence microscope (Olympus, Tokyo, Japan) to determine the transduction efficiency. Western blot and qRT-PCR were used to examine the level of RUNX1 expression. After RUNX1 knockdown efficiency was determined, the cell lines were used to perform further experiments. 


\section{Reverse transcription and quantitative real-time PCR}

A two-step real-time quantitative RT-PCR was used to examine specific mRNA levels. For qRT-PCR analysis, total RNAs were prepared by RNA preparation kit (TransGen, Beijing, China). Reverse transcription was carried out with HiScript Q Select RT SuperMix for qPCR (+gDNA wiper) (Vazyme, Nanjing, China). The primers of RT-PCR were designed using PrimerQuest Tool. The sequences of primers for $\beta$-Actin, RUNX1, IFNB1, ISG15, MxA, TRAF3, RIG-I, MAVS, TBK1, IRF3, STAT1, PR8-M, and PR8-NP are listed in Table 1. Each gene was amplified in triplicate and mean threshold $(\mathrm{Ct})$ values were calculated. The housekeeping gene $\beta$-Actin was used for normalization in gene expression analysis. Relative fold changes in gene expression among groups were determined using the $2^{-\Delta \Delta C t}$ method.

\section{Western blot analysis}

Cells were collected and washed with PBS, and then lysed with RIPA lysis buffer (Beyotime, Shanghai, China) containing a protease inhibitor cocktail (Roche, Basel, Switzerland). The cell lysates were centrifuged at 12,000 $g$ for

Table 1 Primers used for qPCR

\begin{tabular}{|c|c|c|}
\hline Target gene & Direction & Sequence $\left(5^{\prime}-3^{\prime}\right)$ \\
\hline$\beta$-Actin & Forward & ATCTGGCACCACACCTTCTACAATGAGCTGCG \\
\hline$\beta$-Actin & Reverse & CGTCATACTCCTGCTTGCTGATCCACATCTG \\
\hline RUNX1 & Forward & CTTTCAAGGTGGTGGCCCTA \\
\hline RUNX1 & Reverse & CTTGCGGTGGGTTTGTGAAG \\
\hline$|F N B|$ & Forward & TTGTTGAGAACCTCCTGGCT \\
\hline$|F N B|$ & Reverse & TGACTATGGTCCAGGCACAG \\
\hline ISG15 & Forward & CGCAGATCACCCAGAAGATCG \\
\hline ISG15 & Reverse & TTCGTCGCATTTGTCCACCA \\
\hline$M \times A$ & Forward & GTTTCCGAAGTGGACATCGCA \\
\hline$M \times A$ & Reverse & GAAGGGAACTCCTGACAGT \\
\hline TRAF3 & Forward & GCGTGTCAAGAGAGCATCGTT \\
\hline TRAF3 & Reverse & GCAGATGTCCCAGCATTAACT \\
\hline$R / G-I$ & Forward & ACGCAGCCTGCAAGCCTTCC \\
\hline$R I G-I$ & Reverse & TGTGGCAGCCTCCATTGGGC \\
\hline MAVS & Forward & CAGGCCGAGCCTATCATCTG \\
\hline MAVS & Reverse & GGGCTTTGAGCTAGTTGGCA \\
\hline TBK1 & Forward & GGCGGCTAGAAGAGGCTTTG \\
\hline TBK1 & Reverse & CTCCGTCAGCTCGGTGTAG \\
\hline IRF3 & Forward & GCAGGAGGATTTCGGAATCTTC \\
\hline IRF3 & Reverse & GGAAATTCCTCTTCCAGGTTGG \\
\hline STAT1 & Forward & TGGCCCTAAAGGAACTGGAT \\
\hline STAT1 & Reverse & CACTATCCGAGACACCTCGTC \\
\hline PR8-NP & Forward & TGCCTGTGTGTATGGACCTG \\
\hline PR8-NP & Reverse & TTCAAATGCGGCAGAATGGC \\
\hline PR8-M & Forward & CCAGCATCGGTCTCATAGGC \\
\hline PR8-M & Reverse & TCGATCCAGCCATTTGCTCC \\
\hline
\end{tabular}

10 min at $4{ }^{\circ} \mathrm{C}$ and the supernatant was collected. Equal amounts of protein samples were subjected to SDSpolyacrylamide gel electrophoresis and transferred to PVDF membrane (Millipore, Darmstadt, Germany). The membrane was probed with various primary antibodies as indicated and detected using the ECL system with alkaline phosphatase-conjugated secondary antibodies according to the manufacturer's protocol.

\section{Statistical analysis}

All data were expressed as mean $\mathrm{x} \pm$ standard deviation (SD) from at least three independent experiments. The statistical analyses were performed with a two-tailed Student's $t$-test and a two-way ANOVA test. $p<0.05$ was considered statistically significant. Differences between groups were considered significant if $* p<0.05$, highly significant if $* * 00.01$ and extremely significant if *** $p<0.001$.

\section{Results}

\section{RUNX1 is induced by IAV PR8 infection in A549 cells}

We first examined whether IAVs could induce RUNX1 expression. A549 cells were infected with PR8 at MOI of $0.1,1$, and 5 , and the expression of RUNX1 was detected by Western blot and qRT-PCR at $12 \mathrm{~h}$ post-infection (h.p.i.). Increases of RUNX1 protein and mRNA were observed in PR8-infected samples, and the elevation was dose-dependent (Fig. 1a, b). We also examined RUNX1 expression in A549 cells at different time points after PR8 infection. A549 cells were infected with PR8 at an MOI of 1 and were collected at 3, 6, and 12 h.p.i.. The results from the Western blot and qRT-PCR experiments showed that RUNX1 protein synthesis gradually increased and the mRNA level significantly increased about 1.5-fold at 12 h.p.i. $(p<0.01)$ (Fig. 1c, d). RUNX3, a developmental regulator and tumor suppressor that belongs to RUNX family, was induced by IAV H1N1 and H3N2, influenza viral RNA, a synthetic analog of viral double-stranded RNA (dsRNA) polyinosinic-polycytidylic acid (poly(I:C)) in the normal human bronchial epithelial cell line BEAS2B [36]. But RUNX1 was not induced by poly(I:C) in A549 cells (Fig. 1e). Taken together, these data demonstrated that PR8 infection induced RUNX1 expression.

\section{RUNX1 knockdown reduced virus replication}

The aforementioned data showed that H1N1 PR8 viruses infection induced RUNX1 expression in A549 cells, so we hypothesized that RUNX1 may play a role in influenza virus infection. To test this hypothesis, RUNX1 was knocked down in A549 cells by transduction of lentiviruses (Fig. 2a). Results from Western blot and qRT-PCR showed that the level of RUNX1 protein and mRNA was significantly reduced (Fig. 2b, c). 


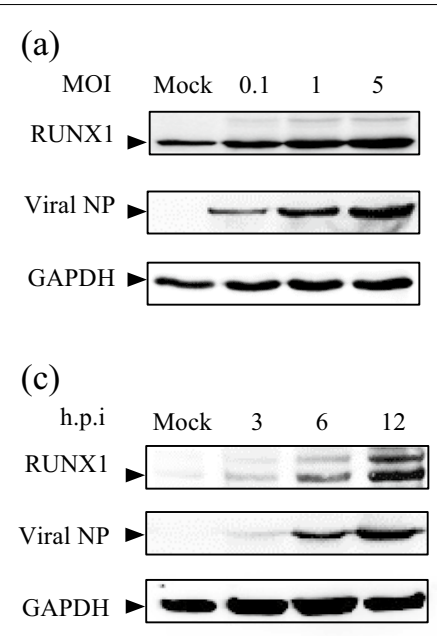

(e)

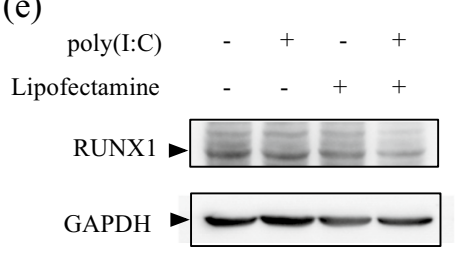

Fig. 1 Influenza virus A/Puerto Rico/8/34 (H1N1) (PR8) infection induced RUNX1 expression. a A549 cells were infected with PR8 at indicated MOls and harvested at 12 h.p.i., protein levels of RUNX1, vial NP, and GAPDH were analyzed by Western blot. b A549 cells were infected with PR8 at indicated MOls and harvested at 12 h.p.i., and RUNX1 and GAPDH mRNA levels were analyzed by qRT-PCR. c A549 cells were infected with IAV at an $\mathrm{MOI}$ of 1 . Cells were harvested at 3, 6, and 12 h.p.i. and analyzed by Western blot with anti-RUNX1, anti-NP, and anti-GAPDH antibodies. d A549 cells were infected with PR8 at an MOI of 1 . Cells were harvested at 3, 6, and 12 h.p.i. and RUNX1 and GAPDH mRNA levels were analyzed by qRT-PCR. e A549 cells were treated with $1 \mu \mathrm{g} / \mathrm{ml}$ poly $(\mathrm{l}: \mathrm{C})$ with $(+)$ or without (-) transfection reagent lipofectamine 2000 for $12 \mathrm{~h}$ and collected for Western blot analysis of RUNX1. Data are mean \pm SD of three independent experiments. Significance is by two-way ANOVA test; ${ }^{*} p<0.05,{ }^{* *} p<0.01$

Next, we examined the progeny virus production in the shRUNX1 and shControl cells infected with PR8 at an MOI of 1 , and harvested the cells at $0,3,6$, and 9 h.p.i. and examined viral NP and M1 protein by Western blot and qRT-PCR. In comparison with the control cells, knockdown of RUNX1 led to a significant reduction of viral M1 protein expression and slight reduction of viral NP protein expression (Fig. 2d), and the RNA of NP and $M$ decreased to $60 \%$ correspondingly in shRUNX1 cells at 8 h.p.i (Fig. 2e). Also, we examined the infectious progeny virus titers in the supernatant collected from shRUNX1 and shControl cells infected with PR8 at an MOI of 0.01. The results showed that virus titer in shRUNX1 cells supernatant was about 17-fold lower than that in shControl cells after $24 \mathrm{~h}$ infection (Fig. 2f). These results demonstrated that knockdown of RUNX1 had an inhibitory effect on the PR8 virus life cycle.

\section{RUNX1 overexpression facilitates IAV replication}

To validate the results that knockdown of RUNX1 impaired IAV PR8 progeny virus production, we performed similar experiments using the A549 cells which transiently overexpressed RUNX1 and the A549 cells which were transfected with an empty vector to serve as the control. Overexpression of RUNX1 was confirmed by Western blot and qRT-PCR at 24 h.p.i. (Fig. 3a, b). To examine the effect of RUNX1 overexpression on IAV replication, we applied Western blot and qRT-PCR to detected viral NP and $M$ in the infected cells. In consistent with the observations we saw in the shRUNX1 cells, more viral NP and M production was found in the cells with RUNX1 overexpression (Fig. 3c, d). To further confirm the impact of RUNX1 on IAV replication, we collected the supernatant and titrated the viruses by $\mathrm{TCID}_{50}$ assay. The results showed that viral titer of PR8 in RUNX1-overexpressing cells was also increased $\sim 18$ fold in comparison with that in control cells (Fig. 3e). Collectively, these results demonstrated that RUNX1 enhanced PR8 infection and progeny virus production in host cells.

\section{RUNX1 negatively regulates IAVs-induced IFN- $\beta$ and ISGs expression}

Our results have shown that RUNX1 regulates IAV PR8 infection. Interferons are the first line of defense against viral infection and IFN- $\beta$ plays key roles in anti-IAVs infection [5, 37]. It is also demonstrated that RUNX1 negatively regulates innate immune responses during viral infection [26]. We next investigated the effects of RUNX1 on IFN- $\beta$ expression upon IAV infection. We performed qRT-PCR to compare the mRNA levels of IFNB1 between RUNX1-knockdown and control cells after PR8 infection. shRUNX1 A549 cells and shControl A549 cells were infected with PR8 at an MOI of 5 , and then the cells were collected at $0,3,6$, h.p.i. Compared with shControl cells, the IFNB1 mRNA levels in the shRUNX1 cells significantly increased upon PR8 infection, and increased fold is $\sim 4$ at $9 \mathrm{~h}$ post PR8 infection (Fig. 4a). The results revealed that knockdown of RUNX1 in A549 cells augmented IAV-induced IFN- $\beta$ expression. To further gain insight into the antiviral state, we also analyzed the mRNA levels of $M x A$ and ISG15, which have been reported to be involved in IAV infection [38-41]. The results showed that the expression of $M x A$ and $I S G 15$ increased $3 \sim 6$ fold in shRUNX1 A549 cells as compared with that in control cells (Fig. 4c, e). We also validated the results by overexpression of RUNX1 in the A549 cells. Accordingly, 
(a)

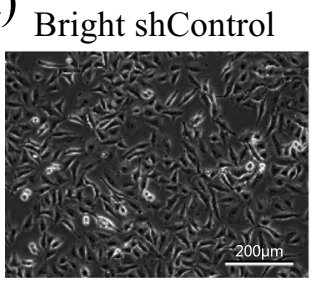

Bright shRUNX1

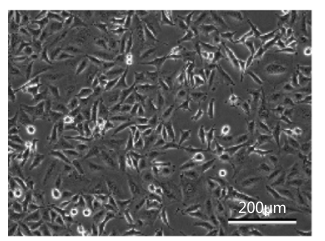

GFP shControl

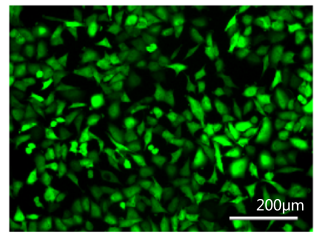

GFP shRUNX1

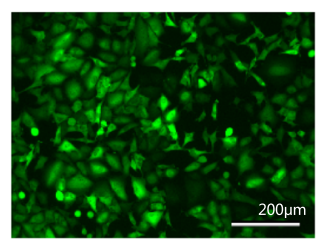

(b)

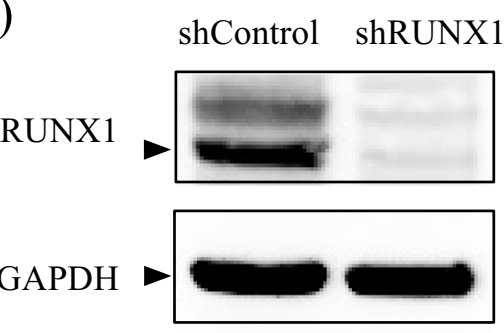

(c)

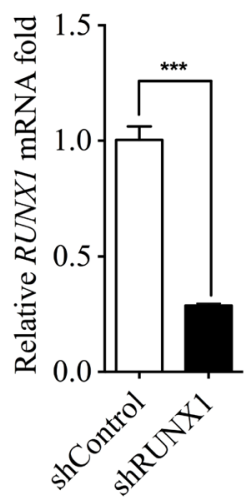

(e)

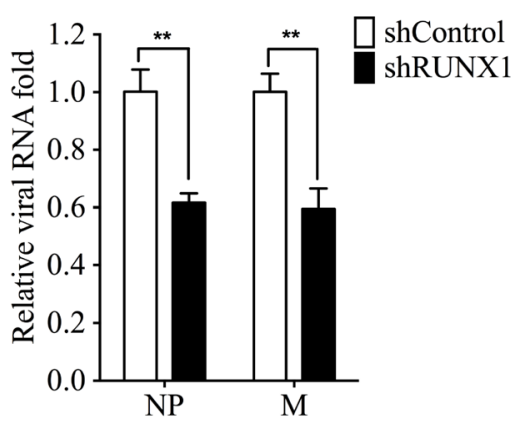

(f)

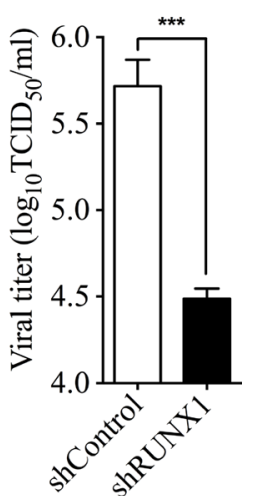

Fig. 2 Knockdown of RUNX1 inhibits PR8 replication. a Stable transfection of A549 cells with RUNX1 shRNA. Bright-field microscopy of and fluorescent microscopy of cells transfected with shControl and shRUNX1 were applied to check the cell growth and GFP expression. $\mathbf{b}$ The RUNX1 protein was assessed by Western blot. (c) The mRNA of RUNX1 was assessed by qRT-PCR. $\mathbf{d}$ The shRUNX1 cells and shControl cells were infected with PR8 at an $\mathrm{MOI}$ of 1 and collected at 3, 6, and 9 h.p.i.. The viral NP and M1 protein were assessed by Western blot. e The shRUNX1 cells and shControl cells were infected with PR8 at an MOI of 1 and collected at 8 h.p.i.. The RNA of viral NP and M was assessed by qRT-PCR. $\mathbf{f}$ The shRUNX1 cells and shControl cells were infected with PR8 $(\mathrm{MOI}=0.01)$ and the culture supernatants were collected at 24 h.p.i. for viral titration by $\mathrm{TCID}_{50}$ assay. All images were captured at $\times 200$ magnification, scale bars $=100 \mu \mathrm{m}$. Data are mean \pm SD of three independent experiments. Significance is by unpaired T-test; ${ }^{* *} p<0.01 .{ }^{* * *} p<0.001$

cells transfected with RUNX1 expression plasmids or vector-only plasmids were infected with PR8 at an MOI of 5, and the cells were collected at 0, 3, 6, 9 h.p.i. for qRT-PCR analysis of IFNB1, MxA and ISG15. Similarly, overexpression of RUNX1 significantly reduced IFNB1, $M x A$, and ISG15 mRNA levels upon PR8 infection (Fig. 4b, d, f). These data indicated that RUNX1 may negatively regulate IAV-induced IFN- $\beta$ responses.

To further investigate whether RUNX1 negatively regulate IFN- $\beta$ signaling upon IAV infection in A549 cells is not strain specific, we infected the A549 cells with influenza A/Zhejiang/163/2020 (H3N2) (ZJ163), A/swine/Jiangsu/C1/2008 (H9N2) (JSC1) and A/ California/04/2009(H1N1) (CA04) at MOI of 1 , and then examined the IFNB1, MxA, and ISG15 expression at 9 h.p.i. We found that the expression of IFNB1, $M x A$, and ISG15 increased in the RUNX1 knockdown cells by $2 \sim$ ninefold compared to the control cells (Fig. 4g).

Interferons and ISGs are very crucial in the antiviral immunity. To test whether RUNX1 could directly regulate IFN- $\beta$ signaling, we analyzed the expression of IFNB1, MxA and ISG15 in the A549 cells without viral infection. As shown in Fig. 4h, i, the expressions of IFNB1, MxA and ISG15 did not altered no matter RUNX1 was knocked down or overexpressed. It suggested that RUNX1 does not directly regulate IFN- $\beta$ and ISGs expression. 


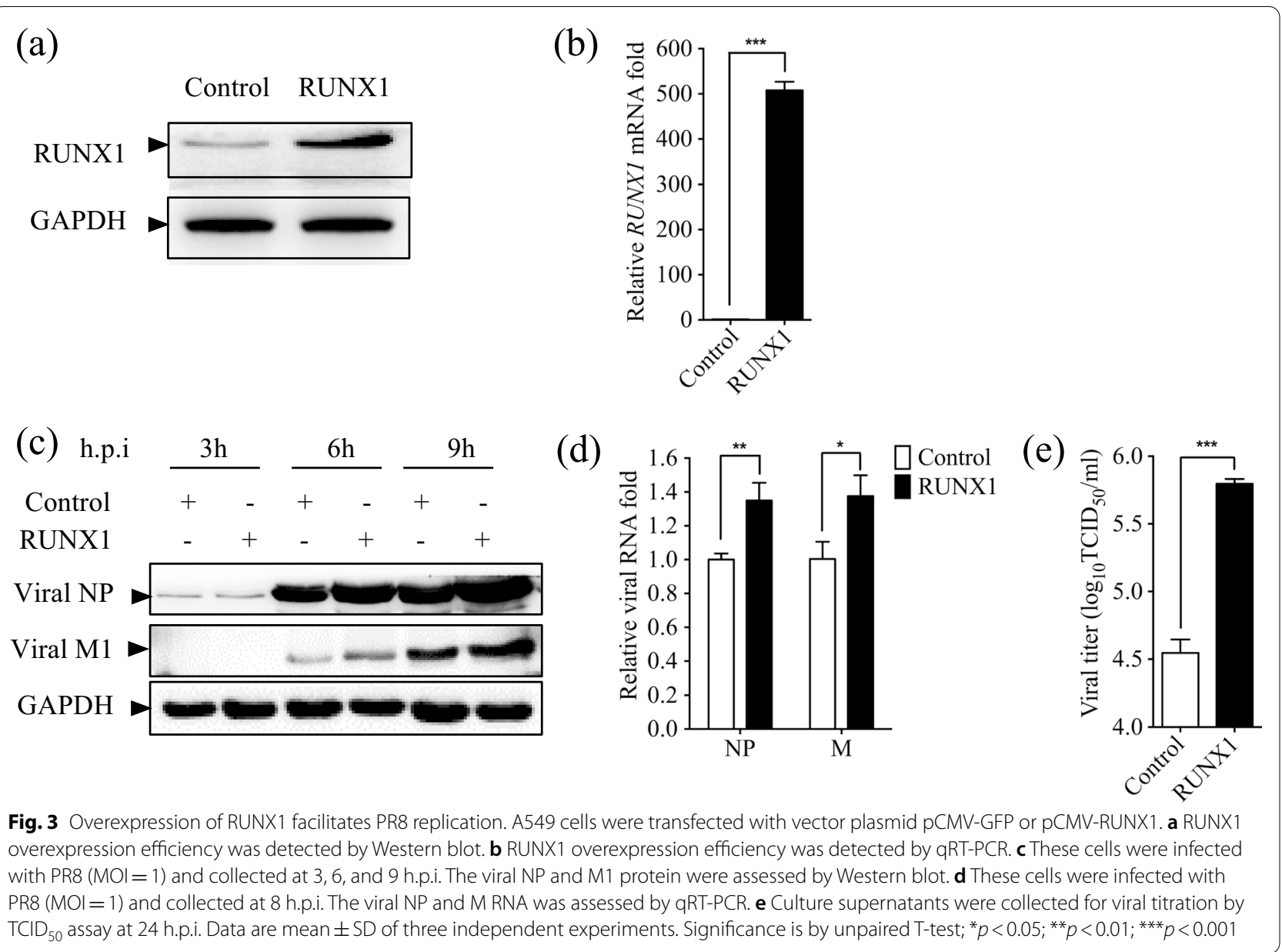

\section{RUNX1 suppresses IRF3- and STAT1-mediated signaling pathways}

In view of the incapability of RUNX1 in direct regulation of IFN- $\beta$ and ISGs expression, we further elucidate the molecular mechanism that how RUNX1 regulates IFN- $\beta$ production, we first analyzed the expression of type I IFN signaling-related genes (TRAF3, RIG-I, MAVS, TBK1, IRF3, and STAT1) in A549 cells by qRT-PCR [6, $27,42,44]$. Results from qRT-PCR assay showed that the mRNA levels of IRF3 and STAT1, whose phosphorylation is important in IFN- $\beta$ and ISGs production $[5,9,42,43]$, were higher in the A549 cells with RUNX1 knockdown (Fig. 5a), while their level decreased in the A549 cells with RUNX1 overexpression (Fig. 5b). Consistently, knockdown of RUNX1 increased IRF3 and STAT1 protein levels (Fig. 5c) and overexpression of RUNX1 decreased IRF3 and STAT1 protein levels (Fig. 5d). Phosphorylation of IRF3 and STAT1 is essential in the activation of IFN- $\beta$ and ISGs expression [9, 43, 45, 46]. We also tested the role of RUNX1 on phosphorylation of IRF3 and STAT1

\footnotetext{
(See figure on next page.)

Fig. 4 RUNX1 attenuates PR8-induced IFN- $\beta$ and ISG expression. $\mathbf{a}, \mathbf{c}$, e The shRUNX1 cells and shControl cells were infected with PR8 (MOI= 5) and harvested at 3, 6, and 9 h.p.i. The mRNA level of IFNB1, MXA and ISG15 was assessed by qRT-PCR. b, d, fThe A549 cells which were transfected with empty plasmid PCMV-GFP or PCMV-RUNX1. After $24 \mathrm{~h}$ transfection, the cells were infected with PR8 (MOI=5) and harvested at 3, 6, and 9 h.p.i. The mRNA level of IFNB1, MXA, and ISG15 was assessed by qRT-PCR. (g) The shRUNX1 cells and shControl cells were infected with influenza A/Zhejiang/163/2020 (H3N2) (ZJ163), A/swine/Jiangsu/C1/2008 (H9N2) (JSC1) and A/California/04/2009(H1N1) (CA04) (MOI=1) and harvested at 9 h.p.i. The mRNA level of IFNB1, MXA and ISG15 in the A549 cells was assessed by qRT-PCR. $\mathbf{h}$ The mRNA level of IFNB1, MXA, and ISG15 in the shRUNX1 cells and shControl cells without viral infection was assessed by qRT-PCR. i The mRNA level of IFNB1, MXA, and ISG15 in the A549 cells which were transfected with empty plasmid pCMV-GFP or pCMV-RUNX1 was assessed by qRT-PCR. Data are mean \pm SD of three independent experiments. Significance is by unpaired T-test; ${ }^{*} p<0.05 ;{ }^{* *} p<0.01$. ${ }^{* *} p<0.001$
} 
(a)

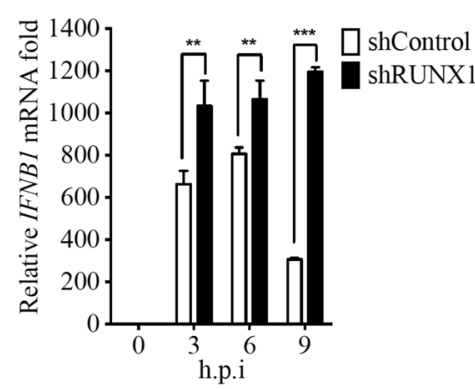

(c)

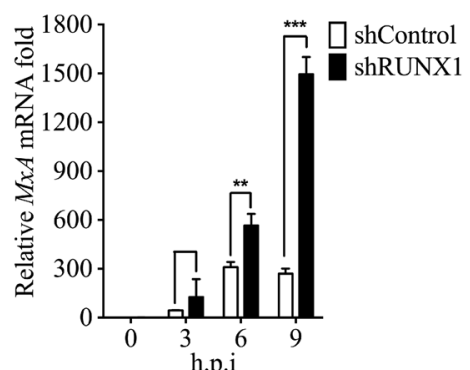

(e)

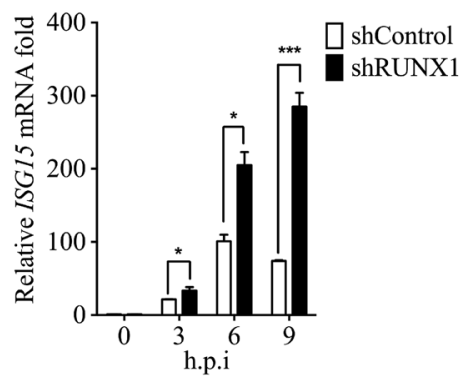

(b)

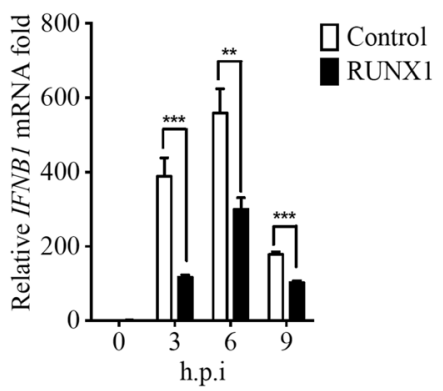

(d)

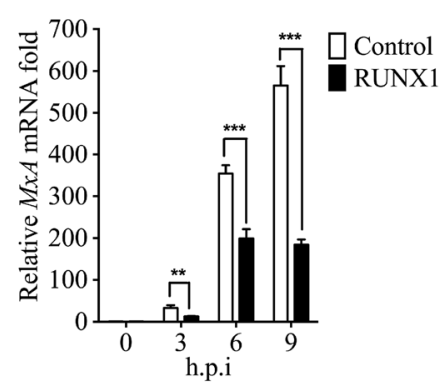

(f)

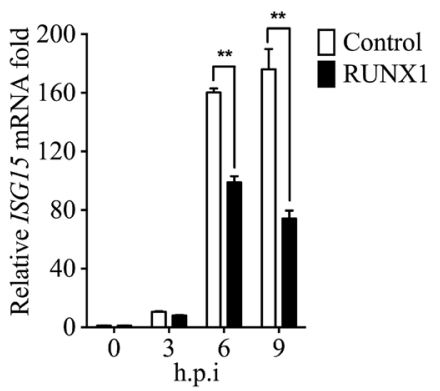

(g)

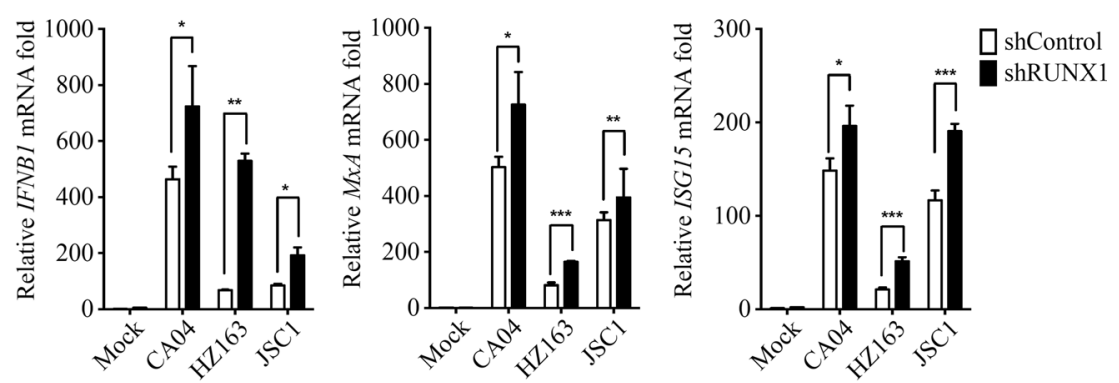

(h)

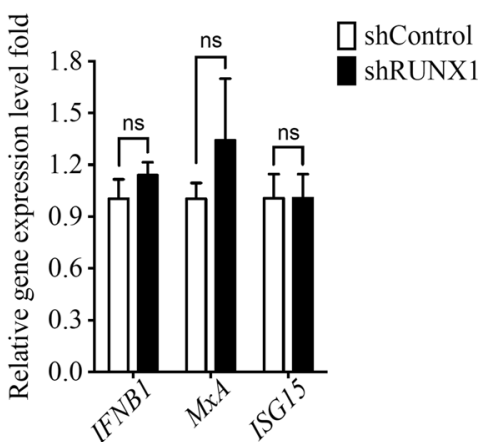

(i)

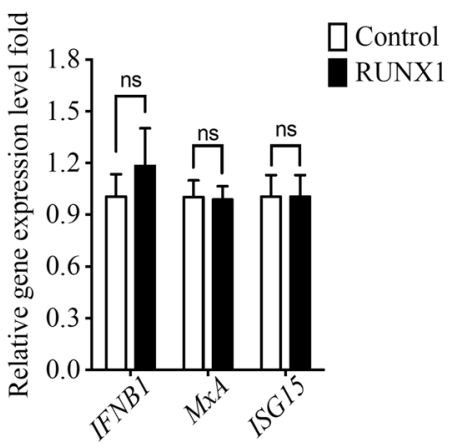


(a)

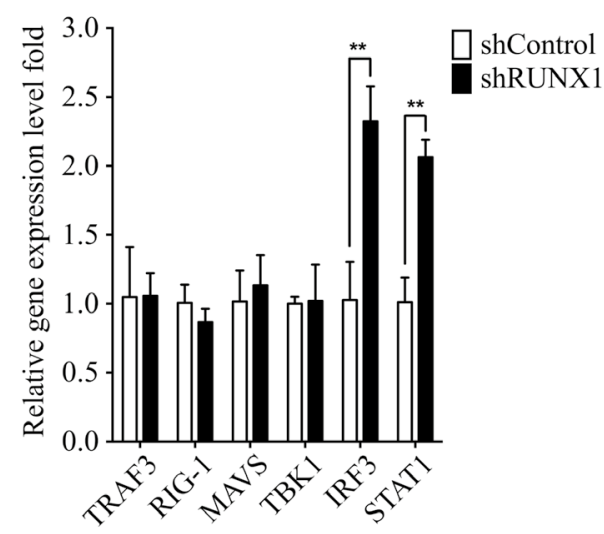

(c)

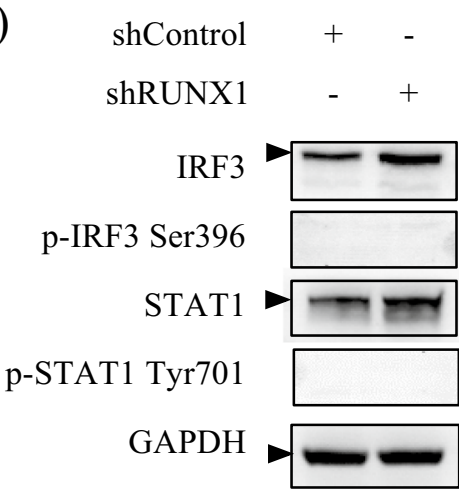

(e)

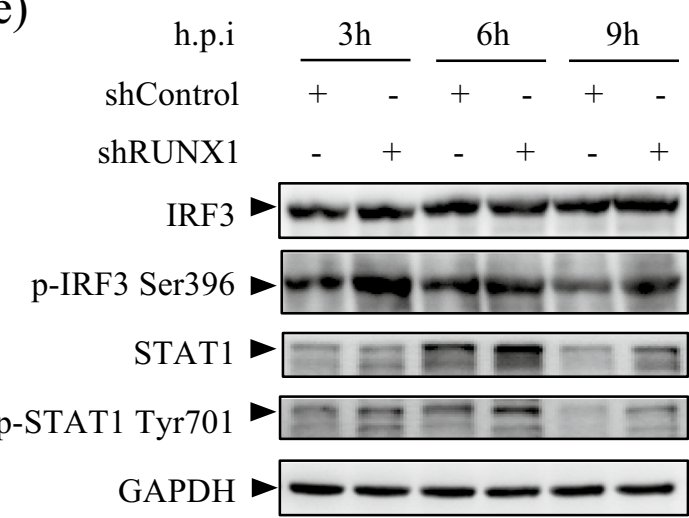

(b)

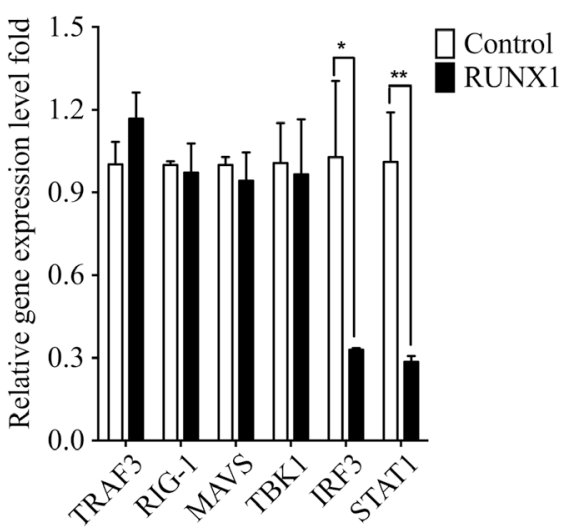

(d)

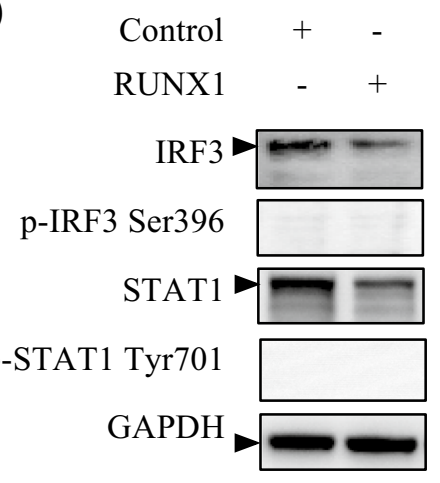

(f)

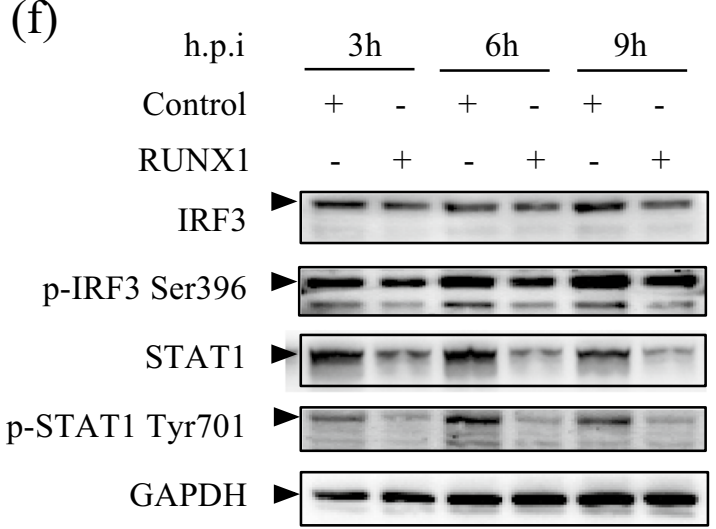

Fig. 5 RUNX1 attenuated IRF3 and STAT1 signaling. a The mRNA level of TRAF3, RIG-I, MAVS, TBK1, IRF3, and STAT1 in the shRUNX1 and shControl cells were assessed by qRT-PCR. $\mathbf{b}$ The mRNA of these genes in A549 cells that were transfected with empty plasmid pCMV-GFP or pCMV-RUNX1 was assessed by qRT-PCR. $\mathbf{c}$, d The protein and the phosphorylation level of IRF3 and STAT1 of these cells were assessed by Western blot. e, $\mathbf{f}$ These cells were infected with PR8 $(\mathrm{MOI}=5)$ and collected at 3, 6, and 9 h.p.i. The protein and the phosphorylation levels of IRF3 and STAT1 in these cells were assessed by Western blot. Data are mean \pm SD of three independent experiments. Significance is by unpaired T-test; ${ }^{*} p<0.05 ;{ }^{* *} p<0.01$

in non-infected A549 cells to exclude the possibility that RUNX1 would impair the phosphorylation of IRF3 and STAT1 in these cells. We did not detect phosphorylation of IRF3 and STAT1 in A549 cells of RUNX1 knockdown or overexpression without virus infection (Fig. 5c, d).
To address whether RUNX1 could indirectly modify phosphorylation of IRF3 and STAT1 upon viral infection, we infected the cells with PR8 at an MOI of 5, collected the cells at 3,6, and 9 h.p.i. and assessed the phosphorylation of IRF3 and STAT1. As shown in Fig. 5e, 
phosphorylation of IRF3 and STAT1 was enhanced when RUNX1 was knocked down, while phosphorylation of IRF3 and STAT1 were markedly decreased in the cells with RUNX1 overexpression compared with the control cells at 3, 6, and $9 \mathrm{~h}$ post PR8 infection (Fig. 5f). These data demonstrated that RUNX1 inhibited IRF3 and STAT1 signaling.

\section{Discussion}

Due to the relatively small genome and a limited number of protein-coding genes, influenza A viruses have to depend on the host to replicate and complete its life cycle [47]. Several studies have suggested that influenza viruses have evolved ways to hijack host factors and to alter host cell metabolism to facilitate viral replication $[1,48]$. For antiviral drug development, it is highly necessary to identify these cellular factors which modulate IAV replication and pathogenesis. In the present study, we identified RUNX1 is an important transcription factor that facilitates replication of H1N1 PR8. It is interesting to know whether the effects of RUNX1 on IAV infection is widely applied by other influenza virus strains. We found that when the cells were infected with H1N1 CA04, H3N2 ZJ163 and H9N2 JSC1, the expression of the IFNB1, $M x A$, and ISG15 increased in the RUNX1 knockdown cells. It indicated RUNX1 would facilitate H1N1 CA04, H3N2 ZJ163 and H9N2 JSC1 infection as well, because IFN- $\beta$ and ISGs are crucial in the antiviral response. Several studies applied genome-wide screens to identify the cellular factor involved in influenza infection and replication with different cells and different influenza strains, and only two groups who used H1N1 identified RUNX1 is one of the hundreds of targets [31-33, 49-53]. Maybe because RUNX1 expression levels are variable in different cells. Here we found that RUNX1 expression is induced by IAV H1N1 PR8 infection in A549 cells. Gan et al. did not detect increased RUNX1 expression in BEAS-2B cells upon H1N1 PR8 infection [36]. The difference may be resulted from the different cells applied. A549 cells are the tumor cells, while BEAS-2B are the normal human bronchial epithelial cell. The intrinsic activities of genes in different cells may be distinct.

Enormous studies have indicated that transcription factor RUNX1 orchestrates many different aspects of biology, including basic cellular and developmental processes, stem cell biology, tumorigenesis, and immunity $[54,55]$. Besides, RUNX1 also plays role in viral infection. RUNX1 regulates apoptosis during transmissible gastroenteritis virus infection [22]; Runx1 activates polyomavirus DNA replication by stimulating the binding of the viral-encoded replication initiator/helicase, large $\mathrm{T}$ antigen, to its replication origin [24]. RUNX1 also interacts with SARS-CoV accessory protein $3 \mathrm{~b}$ interact RUNX1 to enhance transcription of macrophage inflammatory protein (MIP-1 $\alpha$ ) [20]. However, little is known about the innate immune mechanism regulated by RUNX1 during IAV infection. In this study, we found that RUNX1 expression can be induced with time and the dose-dependent manner by H1N1 PR8 infection in A549 cells. But we do not know exactly how IAV induced RUNX1 expression. Many studies suggest that RUNX1 functions as a key player in the replication of various viruses, such as polyomavirus [24], HIV [56], and alphaherpesvirus [57]. Gaur et al. found IAV NA protein interacts with RUNX1 [34]. Our previous study showed that type I and type II IFNs may regulate RUNX1 expression in the embryonic aorta [58]. Upon IAV infection, viral RNA could be rapidly recognized by the PRRs, and type I IFNs was rapidly induced in the cells (Fig. 4a); nevertheless, we did not detect increased RUNX1 expression in A549 cells when treated with poly(I:C), which is a strong type I IFN inducer. Our result was consistent with what Gan et al. observed in BEAS-2B. The results suggested that RUNX1 induction might rely on some steps in the IAV life cycle. The mechanisms are worth future studies.

Our study has also addressed that RUNX1 may facilitate IAV infection by modulating host innate immune systems. We observed that when RUNX1 was knocked down, IAV replication was impaired along with significantly increased IFN- $\beta$ expression. While overexpression of RUNX1 led to the opposite consequences. These findings suggested that RUNX1 attenuated the IFN- $\beta$ production in epithelial cells upon IAV infection. However Gaur et al. recently reported that decreased expression of IFN- $\beta$ in IAV infected U937 cells where AML1 was knocked down using siRNA [34]. These contradictory observations might be due to the different type of cells used. Previous studies have shown that RUNX1 plays an opposite role in the regulation of inflammatory response in the epithelial cell and macrophages in the lung $[15,17$, 59]. These results served as a reminder that RUNX1 may play a dual role in the different cells in the lungs from IAV infected patients. Our results also provided new insights into the role of RUNX1 in regulating immune function in pulmonary disease caused by IAV infection. Furthermore, we examined the effect of RUNX1 on IFN downstream effectors, such as ISG15 and MxA. Overexpression of RUNX1 significantly impaired IAV-induced ISGs expression and knockdown of RUNX1 weakened this inhibition. However, the expression of IFNB1, MxA and ISG15 did not changed in the non-infected A549 cells no matter we knocked down or overexpressed RUNX1. These results indicated that IAV-induced RUNX1 antagonized the innate immunity response by suppressing IAV-induced expression of IFN- $\beta$ and ISGs, 


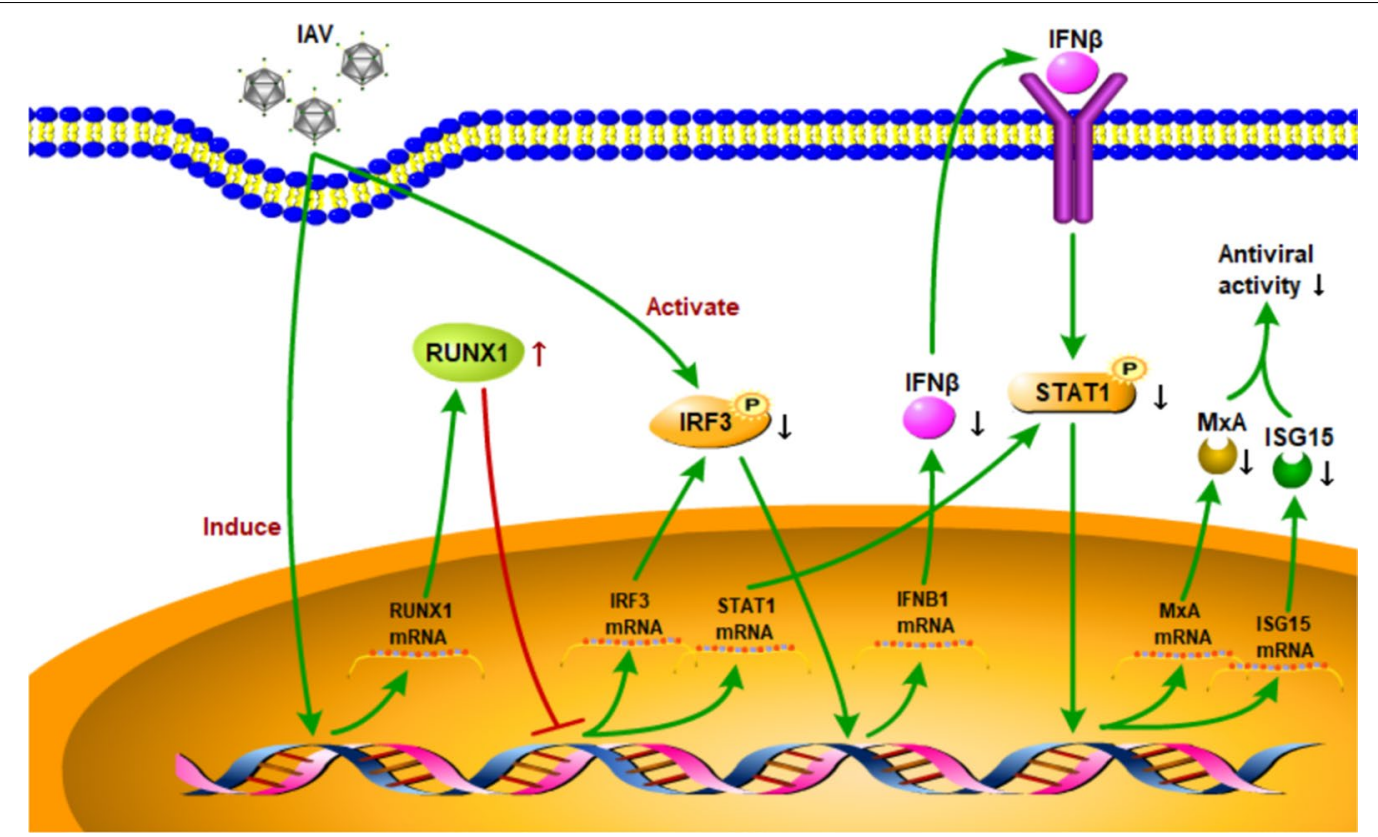

Fig. 6 RUNX1 inhibits the antiviral immune response against IAV by attenuating type I interferon signaling. RUNX1 expression was induced by IAV infection. And then RUNX1 attenuates type I interferon signaling to impede the antiviral immune response

and RUNX1 could not directly regulate IFN- $\beta$ and ISG expression.

To further explore the mechanism by which RUNX1 regulates the IFN signaling, we examined the effect of RUNX1 on the expression of IFN signaling pathwayrelated proteins (TRAF3, RIG-I, MAVS, TBK1, IRF3, STAT1). We observed that overexpression of RUNX1 inhibited IRF3 and STAT1 expression, while knockdown of RUNX1 increased IRF3 and STAT1 expression in A549 cells, no matter whether the A549 cells were infected by IAV or not. Correspondingly, the level of phosphorylated IRF3 and STAT1 changed in the same way in the cells upon IAV infection. Based on the level changed as reflected by Western blot, we supposed that the changed levels of IRF3 and STAT1 phosphorylation in RUNX1-knockdown or overexpression A549 cells during PR8 infection are mainly due to the changed IRF3 and STAT1 expression. Previous studies have reported that IAVs inhibited IRF3 and STAT1 signal transduction by utilizing a variety of host proteins, such as PGRN and A20, to block IFN signaling transduction [60, 61]. Maybe RUNX1 aid IAV infection by impairment of IFN- $\beta$ and ISGs production through inhibition of STAT1 and IRF3. As a DNA-binding transcription factor that regulates genes expression, RUNX1 must gain access to its binding sites within a chromatin context, and then it recruits many other coactivators to promote gene expression [62]. In addition, RUNX1 can serve as transcription repressor by recruiting corepressors to target genes. For example,
RUNX1 binds to corepressor SIN3A complex [63] and the Groucho/TLE repressor complex [64, 65]. Whether RUNX1 active or suppress gene expression, it depends on the cellular and promoter context. Our results in this study indicated that RUNX1 negatively regulates IRF3 and STAT1. To find out whether RUNX1 could directly regulate IRF3 and STAT1 expression, we searched the RUNX1 binding site in the DNA by CHIP-Atlas database (http://chip-atlas.org), and found that RUNX1 can bind many regions located in promoters and gene bodies of STAT1 (chr2:191013544-191014873 and 190979768190981151) and IRF3 (chr19:49665448-49666448, 49664233-49665075, 49661796-49662665, and 49658977-49659365), as well as some regions upstream or downstream $10 \mathrm{~K}$ far away from gene bodies. It is worthwhile to study further by using ChIP-Seq to examine the histone modifications (H3K27me3) in chromatin, or by CO-IP to examine the interaction of RUNX1 and compressor complex, and by molecular method to verify the binding of RUNX1 to the regulatory DNA of STAT1 or IRF3 suppresses gene expression. Also, members of the RUNX family are often in contact with the STAT1 [66-68]. However, STAT1 was known to only act as an upstream effector of RUNX1 so far. For example, STAT1 regulates megakaryopoiesis by altering the expression of RUNX1 [69]. In the antiviral innate response, VSV infection significantly induced downregulation of miR-27a through the IFN/JAK/STAT1/RUNX1 signaling pathway in macrophages to inhibit type I IFN production 
[26]. Our study is the first report that the expression of STAT1 is regulated by RUNX1, which is likely related to the specific regulatory role of RUNX1 in lung epithelial cells. The mechanism of how IAV infection induces RUNX1 expression and how RUNX1 regulates the IFN signaling pathway is still elusive, which requires further investigation in the future. Besides, as a transcription factor, RUNX1 is functionally associated with the immune system development and critical for inducing the production of many immune genes, which suggests RUNX1 may also be involved in other signaling pathways during influenza infection. It is also an interesting direction in our future research.

\section{Conclusion}

In summary, our study demonstrated that induction of RUNX1 expression by IAV infection helped IAV to escape host anti-viral response (Fig. 6). These findings provide a novel insight that RUNX1 may play a key regulatory role in innate immunity during virus infection.

\section{Abbreviations}

IAV: Influenza A virus; PR8: Influenza virus A/Puerto Rico/8/34 (H1N1); ZJ163: Influenza A/Zhejiang/163/2020 (H3N2); JSC1: Influenza A/swine/Jiangsu/ C1/2008 (H9N2); CA04: Influenza A/California/04/2009(H1N1); MOI: Multiplicity of infection; IFN: Interferon; IFN- $\beta$ : Interferon $\beta$; IRF3: Interferon regulatory factor 3; NP: Nucleocapsid protein; M1: Matrix protein 1; ISG: Interferon stimulated gene.

\section{Acknowledgements}

We would like to thank Dr. Jiyong Zhou for kindly providing the antibodies to influenza A virus NP and M1 proteins.

\section{Authors' contributions \\ $\mathrm{YH}$ and QP designed the study, performed the experiments, and analyzed the data, wrote and edited the manuscript. $K Z$, YL and HW performed the experiments. YL conceived and designed the study, verified the data, wrote and edited the manuscript. All authors have read and approved the final manuscript.}

\section{Funding}

The research was supported by the Hainan Provincial Joint Project of Sanya Yazhou Bay Science and Technology City (820LH005), the National Natural Science Foundation of China (31872837) and the Fundamental Research Funds for the Central Universities (2019QNA6026).

\section{Availability of data and materials}

All data generated or analyzed during this study are included in this published article.

\section{Declarations}

\section{Ethics approval and consent to participate}

Not applicable. This article does not contain any studies with human or animal subjects performed by any of the authors.

\section{Consent for publication}

Not applicable.

\section{Competing interests}

The authors declare that they have no competing interests.

\section{Author details}

${ }^{1}$ Department of Veterinary Medicine and Institute of Preventive Veterinary Sciences, Zhejiang University College of Animal Sciences, Hangzhou 310058, Zhejiang, China. ${ }^{2}$ Zhejiang Provincial Key Laboratory of Preventive Veterinary Medicine, Hangzhou 310058, Zhejiang, China. ${ }^{3}$ Hainan Institute, Zhejiang University, Sanya 572025, Hainan, China.

Received: 13 August 2021 Accepted: 14 February 2022

Published online: 05 March 2022

\section{References}

1. Long JS, Mistry B, Haslam SM, Barclay WS. Host and viral determinants of influenza A virus species specificity. Nat Rev Microbiol. 2019;17(2):67-81.

2. Petrova VN, Russell CA. The evolution of seasonal influenza viruses. Nat Rev Microbiol. 2018;16(1):47-60.

3. Kalil AC, Thomas PG. Influenza virus-related critical illness: pathophysiology and epidemiology. Critical Care (Lond, Engl). 2019;23(1):258.

4. Chen X, Liu S, Goraya MU, Maarouf M, Huang S, Chen JL. Host immune response to influenza A virus infection. Front Immunol. 2018;9:320.

5. Villalon-Letelier F, Brooks AG, Saunders PM, Londrigan SL, Reading PC. Host cell restriction factors that limit influenza A infection. Viruses. 2017;9(12):376

6. Le Goffic R, Pothlichet J, Vitour D, Fujita T, Meurs E, Chignard M, Si-Tahar M. Cutting edge: influenza A virus activates TLR3-dependent inflammatory and RIG-I-dependent antiviral responses in human lung epithelial cells. J Immunol. 2007;178(6):3368-72.

7. Ronni T, Sareneva T, Pirhonen J, Julkunen I. Activation of IFN-alpha, IFN-gamma, MxA, and IFN regulatory factor 1 genes in influenza A virus-infected human peripheral blood mononuclear cells. J Immunol. 1995;154(6):2764-74.

8. Genin P, Vaccaro A, Civas A. The role of differential expression of human interferon-a genes in antiviral immunity. Cytokine Growth Factor Rev. 2009;20(4):283-95.

9. Pauli EK, Schmolke M, Wolff T, Viemann D, Roth J, Bode JG, Ludwig S. Influenza A virus inhibits type I IFN signaling via NF-kappaB-dependent induction of SOCS-3 expression. PLoS Pathog. 2008;4(11):e1000196.

10. Wei H, Wang S, Chen Q, Chen Y, Chi X, Zhang L, Huang S, Gao GF, Chen JL. Suppression of interferon lambda signaling by SOCS-1 results in their excessive production during influenza virus infection. PLoS Pathog. 2014;10(1):e1003845.

11. Mill CP, Fiskus W. RUNX1-targeted therapy for AML expressing somatic or germline mutation in RUNX1. Blood. 2019;134(1):59-73.

12. Haferlach T, Meggendorfer M. More than a fusion gene: the RUNX1RUNX1T1 AML. Blood. 2019;133(10):1006-7.

13. Chen MJ, Yokomizo T, Zeigler BM, Dzierzak E, Speck NA. Runx 1 is required for the endothelial to haematopoietic cell transition but not thereafter. Nature. 2009;457(7231):887-91.

14. Yzaguirre AD, de Bruijn MF, Speck NA. The role of Runx1 in embryonic blood cell formation. Adv Exp Med Biol. 2017;962:47-64.

15. Tang X, Sun L, Jin X, Chen Y, Zhu H, Liang Y, Wu Q, Han X, Liang J, Liu $X$, et al. Runt-related transcription factor 1 regulates LPS-induced acute lung injury via NF-kappaB signaling. Am J Respir Cell Mol Biol. 2017;57(2):174-83.

16. Nakagawa M, Shimabe M, Watanabe-Okochi N, Arai S, Yoshimi A, Shinohara A, Nishimoto N, Kataoka K, Sato T, Kumano K, et al. AML1/RUNX1 functions as a cytoplasmic attenuator of NF-kappaB signaling in the repression of myeloid tumors. Blood. 2011;118(25):6626-37.

17. Luo MC, Zhou SY, Feng DY, Xiao J, Li WY, Xu CD, Wang HY, Zhou T. Runtrelated transcription factor 1 (RUNX1) binds to p50 in macrophages and enhances TLR4-triggered Inflammation and septic shock. J Biol Chem. 2016;291(42):22011-20.

18. Kim DY, Kwon E, Hartley PD, Crosby DC, Mann S, Krogan NJ, Gross JD. CBFbeta stabilizes HIV Vif to counteract APOBEC3 at the expense of RUNX1 target gene expression. Mol Cell. 2013;49(4):632-44.

19. Huan C, Li Z, Ning S, Wang H, Yu XF, Zhang W. Long noncoding RNA uc002yug.2 activates HIV-1 latency through regulation of mRNA levels of various RUNX1 isoforms and increased tat expression. J Virol. 2018;92(9):e01844-e1917. 
20. Varshney B, Agnihothram S, Agnihotram S, Tan Y-J, Baric R, Lal SK. SARS coronavirus $3 \mathrm{~b}$ accessory protein modulates transcriptional activity of RUNX1b. PLOS ONE. 2012;7(1):e29542.

21. Gunnell A, Webb HM, Wood CD, McClellan MJ, Wichaidit B, Kempkes B, Jenner RG, Osborne C, Farrell PJ, West MJ. RUNX super-enhancer control through the Notch pathway by Epstein-Barr virus transcription factors regulates B cell growth. Nucleic Acids Res. 2016;44(10):4636-50.

22. Zhao X, Song X, Bai X, Fei N, Huang Y, Zhao Z, Du Q, Zhang H, Zhang L, Tong D. miR-27b attenuates apoptosis induced by transmissible gastroenteritis virus (TGEV) infection via targeting runt-related transcription factor 1 (RUNX1). PeerJ. 2016:4:e1635.

23. Marshall LJ, Moore AC, Ohki M, Kitabayashi I, Patterson D, Ornelles DA. RUNX1 permits E4orf6-directed nuclear localization of the adenovirus E1B-55K protein and associates with centers of viral DNA and RNA synthesis. J Virol. 2008;82(13):6395-408.

24. Murakami Y, Chen L-F, Sanechika N, Kohzaki H, Ito Y. Transcription factor Runx1 recruits the polyomavirus replication origin to replication factories. J Cell Biochem. 2007;100(5):1313-23.

25. Ito Y. RUNX genes in development and cancer: regulation of viral gene expression and the discovery of RUNX family genes. Adv Cancer Res. 2008;99:33-76

26. Zheng Q, Hou J, Zhou Y, Yang Y, Cao X. Type I IFN-inducible downregulation of MicroRNA-27a feedback inhibits antiviral innate response by upregulating Siglec1/TRIM27. J Immunol. 2016;196(3):1317-26.

27. Iwasaki A, Pillai PS. Innate immunity to influenza virus infection. Nat Rev Immunol. 2014;14(5):315-28.

28. Ludwig S. Disruption of virus-host cell interactions and cell signaling pathways as an anti-viral approach against influenza virus infections. Biol Chem. 2011;392(10):837-47.

29. Lee SM, Yen HL. Targeting the host or the virus: current and novel concepts for antiviral approaches against influenza virus infection. Antiviral Res. 2012;96(3):391-404.

30. Loregian A, Mercorelli B, Nannetti G, Compagnin C, Palu G. Antiviral strategies against influenza virus: towards new therapeutic approaches. Cell Mol Life Sci. 2014;71(19):3659-83.

31. Karlas A, Machuy N, Shin Y, Pleissner KP, Artarini A, Heuer D, Becker D, Khalil H, Ogilvie LA, Hess S, et al. Genome-wide RNAi screen identifies human host factors crucial for influenza virus replication. Nature. 2010;463(7282):818-22.

32. Brass AL, Huang IC, Benita Y, John SP, Krishnan MN, Feeley EM, Ryan BJ, Weyer JL, van der Weyden L, Fikrig E, et al. The IFITM proteins mediate cellular resistance to influenza A H1N1 virus, West Nile virus, and dengue virus. Cell. 2009;139(7):1243-54.

33. Ranaware PB, Mishra A, Vijayakumar P, Gandhale PN, Kumar H, Kulkarni DD, Raut AA. Genome wide host gene expression analysis in chicken lungs infected with avian influenza viruses. PLoS ONE. 2016;11(4):e0153671.

34. Gaur P, Kumar P, Sharma A, Lal SK. AML1 protein interacts with influenza $A$ virus neuraminidase and upregulates IFN-beta response in infected mammalian cells. Lett Appl Microbiol. 2020;70(4):252-8.

35. Wu X, Wang S, Yu Y, Zhang J, Sun Z, Yan Y, Zhou J. Subcellular proteomic analysis of human host cells infected with H3N2 swine influenza virus. Proteomics. 2013;13(22):3309-26.

36. Gan H, Hao Q, Idell S, Tang H. Transcription factor Runx3 is induced by influenza A virus and double-strand RNA and mediates airway epithelial cell apoptosis. Sci Rep. 2015;5:17916.

37. Medina RA, Garcia-Sastre A. Influenza A viruses: new research developments. Nat Rev Microbiol. 2011;9(8):590-603.

38. Manz B, Dornfeld D, Gotz V, Zell R, Zimmermann P, Haller O, Kochs G, Schwemmle M. Pandemic influenza A viruses escape from restriction by human MxA through adaptive mutations in the nucleoprotein. PLoS Pathog. 2013;9(3):e1003279.

39. Gotz V, Magar L, Dornfeld D, Giese S, Pohlmann A, Hoper D, Kong BW, Jans DA, Beer M, Haller O, et al. Influenza A viruses escape from MxA restriction at the expense of efficient nuclear vRNP import. Sci Rep. 2016;6:23138

40. Harty RN, Pitha PM, Okumura A. Antiviral activity of innate immune protein ISG15. J Innate Immun. 2009;1(5):397-404.

41. Hsiang TY, Zhao C, Krug RM. Interferon-induced ISG15 conjugation inhibits influenza A virus gene expression and replication in human cells. J Virol. 2009:83(12):5971-7.
42. Goraya MU, Wang S, Munir M, Chen JL. Induction of innate immunity and its perturbation by influenza viruses. Protein Cell. 2015;6(10):712-21.

43. Mogensen TH. IRF and STAT transcription factors — from basic biology to roles in infection, protective immunity, and primary immunodeficiencies. Front Immunol. 2018;9:3047.

44. Pothlichet J, Meunier I, Davis BK, Ting JP, Skamene E, von Messling V, Vidal SM. Type I IFN triggers RIG-I/TLR3/NLRP3-dependent inflammasome activation in influenza A virus infected cells. PLoS Pathog. 2013;9(4):e1003256.

45. Kuo RL, Zhao C, Malur M, Krug RM. Influenza A virus strains that circulate in humans differ in the ability of their NS1 proteins to block the activation of IRF3 and interferon-beta transcription. Virology. 2010;408(2):146-58.

46. Kuo RL, Li LH, Lin SJ, Li ZH, Chen GW, Chang CK, Wang YR, Tam EH, Gong YN, Krug RM, et al. Role of N terminus-truncated NS1 proteins of influenza A Virus in Inhibiting IRF3 Activation. J Virol. 2016;90(9):4696-705.

47. Watanabe T, Watanabe S, Kawaoka Y. Cellular networks involved in the influenza virus life cycle. Cell Host Microbe. 2010;7(6):427-39.

48. Wang $X$, Hinson ER, Cresswell P. The interferon-inducible protein viperin inhibits influenza virus release by perturbing lipid rafts. Cell Host Microbe. 2007;2(2):96-105

49. König R, Stertz S, Zhou Y, Inoue A, Hoffmann HH, Bhattacharyya S, Alamares JG, Tscherne DM, Ortigoza MB, Liang Y, et al. Human host factors required for influenza virus replication. Nature. 2010;463(7282):813-7.

50. Hao L, Sakurai A, Watanabe T, Sorensen E, Nidom CA, Newton MA, Ahlquist P, Kawaoka Y. Drosophila RNAi screen identifies host genes important for influenza virus replication. Nature. 2008;454(7206):890-3.

51. Sui B, Bamba D, Weng K, Ung H, Chang S, Van Dyke J, Goldblatt M, Duan R, Kinch MS, Li WB. The use of random homozygous gene perturbation to identify novel host-oriented targets for influenza. Virology. 2009;387(2):473-81.

52. Li B, Clohisey SM. Genome-wide CRISPR screen identifies host dependency factors for influenza A virus infection. Nat Commun. 2020;11(1):164.

53. Han J, Perez JT, Chen C, Li Y, Benitez A, Kandasamy M, Lee Y, Andrade J, tenOever B, Manicassamy B. Genome-wide CRISPR/Cas9 screen identifies host factors essential for influenza virus replication. Cell Rep. 2018;23(2):596-607.

54. Ito Y, Bae SC, Chuang LS. The RUNX family: developmental regulators in cancer. Nat Rev Cancer. 2015;15(2):81-95.

55. de Bruijn M, Dzierzak E. Runx transcription factors in the development and function of the definitive hematopoietic system. Blood. 2017;129(15):2061-9.

56. Klase Z, Yedavalli VSRK, Houzet L, Perkins M, Maldarelli F, Brenchley J, Strebel K, Liu P, Jeang K-T. Activation of HIV-1 from latent infection via synergy of RUNX1 inhibitor Ro5-3335 and SAHA. PLoS Pathog. 2014;10(3):e1003997.

57. Xiao K, Xiong D, Chen G, Yu J, Li Y, Chen K, Zhang L, Xu Y, Xu Q, Huang X, et al. RUNX1-mediated alphaherpesvirus-host trans-species chromatin interaction promotes viral transcription. Sci Adv. 2021;7(26):1.

58. Li Y, Esain V, Teng L, Xu J, Kwan W, Frost IM, Yzaguirre AD, Cai X, Cortes $M$, Maijenburg MW, et al. Inflammatory signaling regulates embryonic hematopoietic stem and progenitor cell production. Genes Dev. 2014;28(23):2597-612.

59. Tang $X$, Sun L, Wang G, Chen B, Luo F. RUNX1: a regulator of NF-kB signaling in pulmonary diseases. Curr Protein Pept Sci. 2018;19(2):172-8.

60. Wei F, Jiang Z, Sun H, Pu J, Sun Y, Wang M, Tong Q, Bi Y, Ma X, Gao GF. Induction of PGRN by influenza virus inhibits the antiviral immune responses through downregulation of type I interferons signaling. PLoS Pathog. 2019;15(10):e1008062.

61. Feng W, Sun X, Shi N, Zhang M, Guan Z, Duan M. Influenza a virus NS1 protein induced A20 contributes to viral replication by suppressing interferon-induced antiviral response. Biochem Biophys Res Commun. 2017:482(4):1107-13.

62. Collins A, Littman DR, Taniuchi I. RUNX proteins in transcription factor networks that regulate T-cell lineage choice. Nat Rev Immunol. 2009;9(2):106-15.

63. Lutterbach B, Westendorf JJ, Linggi B, Isaac S, Seto E, Hiebert SW. A mechanism of repression by acute myeloid leukemia-1, the target of multiple chromosomal translocations in acute leukemia. J Biol Chem. 2000;275(1):651-6.

64. Levanon D, Goldstein RE, Bernstein Y, Tang H, Goldenberg D, Stifani S, Paroush Z, Groner Y. Transcriptional repression by AML1 and LEF-1 is 
mediated by the TLE/Groucho corepressors. Proc Natl Acad Sci USA. 1998;95(20):11590-5.

65. Simeoni F, Romero-Camarero I, Camera F, Amaral FMR, Sinclair OJ,

Papachristou EK, Spencer GJ, Lie-A-Ling M, Lacaud G, Wiseman DH, et al. Enhancer recruitment of transcription repressors RUNX1 and TLE3 by misexpressed FOXC1 blocks differentiation in acute myeloid leukemia. Cell Rep. 2021;36(12):109725.

66. Kim S, Koga T, Isobe M, Kern BE, Yokochi T, Chin YE, Karsenty G, Taniguchi T, Takayanagi H. Stat1 functions as a cytoplasmic attenuator of Runx2 in the transcriptional program of osteoblast differentiation. Genes Dev. 2003;17(16):1979-91.

67. Dernowsek JA, Pereira MC, Fornari TA, Macedo C, Assis AF, Donate PB, Bombonato-Prado KF, Passos-Bueno MR, Passos GA. Posttranscriptional interaction between miR-450a-5p and miR-28-5p and STAT1 mRNA triggers osteoblastic differentiation of human mesenchymal stem cells. J Cell Biochem. 2017;118(11):4045-62.

68. Miyazono K, Maeda S, Imamura T. Coordinate regulation of cell growth and differentiation by TGF-beta superfamily and Runx proteins. Oncogene. 2004;23(24):4232-7.

69. Huang Z, Richmond TD, Muntean AG, Barber DL, Weiss MJ, Crispino JD. STAT1 promotes megakaryopoiesis downstream of GATA-1 in mice. J Clin Invest. 2007;117(12):3890-9.

\section{Publisher's Note}

Springer Nature remains neutral with regard to jurisdictional claims in published maps and institutional affiliations.

- fast, convenient online submission

- thorough peer review by experienced researchers in your field

- rapid publication on acceptance

- support for research data, including large and complex data types

- gold Open Access which fosters wider collaboration and increased citations

- maximum visibility for your research: over $100 \mathrm{M}$ website views per year

At BMC, research is always in progress.

Learn more biomedcentral.com/submissions 